\title{
ENERGY THEOREMS FOR MAGNETOELASTIC WAVES IN A PERFECTLY CONDUCTING MEDIUM*
}

\author{
BY \\ GEORGE DASSIOS \\ National Technical University of Athens
}

1. Introduction. Magnetoelastic waves appear in a conducting elastic medium as a result of the interaction of elastic disturbances with electromagnetic waves [10]. In this paper we discuss initially the basic equations and study the magnetoelastic waves in a perfectly conducting medium. As in the case of linear, homogeneous and isotropic elasticity, there exist three mutually orthogonal elastic waves propagating in the medium. Nevertheless, the existence of a constant magnetic field alters the phase velocities as well as the polarization vectors of the three elastic waves. The three phase velocities are all different and only one of the three polarization vectors is orthogonal to the direction of propagation. Therefore there is one transverse wave and the other two waves form arbitrary (in general) angles with the direction of propagation.

In the sequel we prove that the total energy as well as the energy of the transverse wave are conserved.

The question of equipartion of energy is studied next. The asymptotic form of equipartion of energy was first observed by Lax and Phillips [8] and the corresponding finite form by Duffin [5]. Other results on equipartion of energy are discussed in $[1,2,4,6,7$, 9], 11]. In [3] the author proved that for elastic waves with compact initial support the kinetic energy becomes equal to the strain energy after some finite time proportional to the radius of the smallest sphere that contains the support of the initial data. An analogous result is proved in this paper. For magnetoelastic waves in a perfectly conducting, linear, homogeneous and isotropic medium, equipartion occurs between the kinetic and the sum of the strain plus the "interaction" energies. The "interaction energy" is the part of the total energy that depends on the existence of the magnetic field and vanishes when the magnetic field disappears. For initial data with compact support the above form of equipartion of energy is attained in finite time, while for initial data with finite energy the equipartion occurs asymptotically. It is also proved that the same equipartition results are true for the transverse wave whose energy is conserved.

2. Basic equations. Let the space $\mathbb{R}^{3}$ be filled with an isotropic and homogeneous elastic material which can propagate electromagnetic waves as well. Assume that a constant magnetic field $\mathbf{H}$ is applied throughout and that the medium has infinite electric conductivity. According to the general theory of magnetoelasticity [10], the displacement field $\mathbf{u}(\mathbf{x}, t)$ and magnetic field $\mathbf{h}(\mathbf{x}, t)$ satisfy the coupled system of partial differential

\footnotetext{
* Received September 5, 1980. The author wants to express his appreciation to Mr. Demitrios Tjanetis for his help with the long calculations.
} 
equations

$$
\begin{gathered}
\mathbf{h}=\nabla \times(\mathbf{u} \times \mathbf{H})=\mathbf{H} \cdot \nabla \mathbf{u}-\mathbf{H} \nabla \cdot \mathbf{u}, \\
\rho \mathbf{u}_{t t}=\mu \nabla^{2} \mathbf{u}+(\lambda+\mu) \nabla(\nabla \cdot \mathbf{u})+\frac{\mu_{0}}{4 \pi}(\nabla \times \mathbf{h}) \times \mathbf{H},
\end{gathered}
$$

where $\rho$ is the mass density, $\lambda$ and $\mu$ the Lamé constants and $\mu_{0}$ the magnetic permeability of the medium.

Choose a coordinate system $x_{i}, i=1,2,3$, so that $\mathbf{H}=(0,0, H)$ where $H=|\mathbf{H}|$. Let

$$
\hat{\mathbf{u}}(\xi, t)=(2 \pi)^{-3 / 2} \int_{\mathbb{R}^{3}} \mathbf{u}(\mathbf{x}, t) e^{i \mathbf{x} \cdot \xi} d^{3} x
$$

and

$$
\hat{\mathbf{h}}(\xi, t)=(2 \pi)^{-3 / 2} \int_{\mathbb{R}^{3}} \mathbf{h}(\mathbf{x}, t) e^{i \mathbf{x} \cdot \xi} d^{3} x
$$

be the Fourier transforms of $\mathbf{u}$ and $\mathbf{h}$ respectively. By Fourier transforming Eqs. (1) and (2) we obtain

$$
\begin{aligned}
\hat{\mathbf{h}}= & -i r[(\boldsymbol{\alpha} \cdot \mathbf{H}) \hat{\mathbf{u}}-(\boldsymbol{\alpha} \cdot \hat{\mathbf{u}}) \mathbf{H}] \\
\rho \hat{\mathbf{u}}_{t t}= & -\mu r^{2} \hat{\mathbf{u}}-(\lambda+\mu) r^{2} \alpha \boldsymbol{\alpha} \cdot \hat{\mathbf{u}} \\
& -i \frac{\mu_{0} r}{4 \pi}[(\boldsymbol{\alpha} \cdot \mathbf{H}) \hat{\mathbf{h}}-(\mathbf{H} \cdot \hat{\mathbf{h}}) \boldsymbol{\alpha}],
\end{aligned}
$$

where

$$
\xi=r \alpha=r\left(\alpha_{1}, \alpha_{2}, \alpha_{3}\right), \quad|\alpha|=1 .
$$

Substituting $\hat{\mathbf{h}}$ from (5) into (6) and setting

$$
v_{s}^{2}=\frac{\mu}{\rho}, \quad v_{p}^{2}=\frac{\lambda+2 \mu}{\rho}, \quad v_{a}^{2}=\frac{\mu_{0} H^{2}}{4 \pi \rho},
$$

we obtain the system of ordinary differential equations

$$
\frac{d^{2} \hat{\mathbf{u}}}{d t^{2}}+r^{2} \mathbb{M} \cdot \hat{\mathbf{u}}=0
$$

where the dyadic $\mathbb{M}$ is given by

$$
\mathbb{M}=v_{p}^{2} \alpha \alpha+v_{s}^{2}(0-\alpha \alpha)+v_{\alpha}^{2}\left[\alpha \alpha+\alpha_{3}^{2} 0-\alpha_{3}\left(\alpha \mathbf{x}_{3}+\mathbf{x}_{3} \alpha\right)\right] .
$$

Here

$$
0=\mathbf{x}_{1} \mathbf{x}_{1}+\mathbf{x}_{2} \mathbf{x}_{2}+\mathbf{x}_{3} \mathbf{x}_{3}
$$

is the identity dyadic and $\mathbf{x}_{1}, \mathbf{x}_{2}, \mathbf{x}_{3}$ are the base vectors of the Cartesian coordinate system. The term that is proportional to $v_{a}^{2}$ is due to presence of the external magnetic field $\mathbf{H}$. The constants $v_{s}, v_{p}$ and $v_{a}$ represent the phase velocity of a transverse wave, the phase velocity of a longitudinal wave and the Alfvén velocity, respectively.

Let the displacement $\mathbf{u}$ and the velocity $\mathbf{u}_{t}$ have initially compact support, and assume that $R$ is the radius of the smallest sphere that contains the supp $\mathbf{u}_{0}$ and the supp $\mathbf{u}_{1}$, 
where

$$
\mathbf{u}_{0}(\mathbf{x})=\mathbf{u}(\mathbf{x}, 0), \quad \mathbf{u}_{1}(\mathbf{x})=\mathbf{u}_{t}(\mathbf{x}, 0) .
$$

The Fourier transforms of the initial data are given by

$$
\hat{\mathbf{u}}_{0}(\xi)=\hat{\mathbf{u}}(\xi, 0), \quad \hat{\mathbf{u}}_{1}(\xi)=\hat{\mathbf{u}}_{i}(\xi, 0) .
$$

3. Magnetoelastic waves. The following theorem asserts that along each direction there are three plane waves that propagate with different phase velocities. These three waves are mutually orthogonally polarized; one of them is transverse and the other two form an arbitrary angle with the direction of propagation.

THEOREM 1. The solution of the system (11)-(12) that satisfies the initial conditions (16), (17) is given by

$$
\hat{\mathbf{u}}(t)=\sum_{i=1}^{3} \mathbf{w}_{i}(t)=\sum_{i=1}^{3} \mathbf{e}_{i} \mathbf{e}_{i} \cdot\left\lfloor\hat{\mathbf{u}}_{0} \cos \left(\lambda_{i} r t\right)+\hat{\mathbf{u}}_{1} \frac{\sin \left(\lambda_{i} r t\right)}{\lambda_{i} r}\right]
$$

where

$$
\begin{aligned}
\lambda_{1}^{2} & =v_{s}^{2}+v_{a}^{2} \alpha_{3}^{2}, \\
\lambda_{2}^{2} & =\frac{1}{2}\left(v_{p}^{2}+v_{s}^{2}+v_{a}^{2}\right)+\frac{1}{2}\left[\left(v_{p}^{2}-v_{s}^{2}+v_{a}^{2}\right)^{2}-4 v_{a}^{2} \alpha_{3}^{2}\left(v_{p}^{2}-v_{s}^{2}\right)\right]^{1 / 2}, \\
\lambda_{3}^{2} & =\frac{1}{2}\left(v_{p}^{2}+v_{s}^{2}+v_{a}^{2}\right)-\frac{1}{2}\left[\left(v_{p}^{2}-v_{s}^{2}+v_{a}^{2}\right)^{2}-4 v_{a}^{2} \alpha_{3}^{2}\left(v_{p}^{2}-v_{s}^{2}\right)\right]^{1 / 2}, \\
\mathbf{e}_{i} & =\mathbf{c}_{i} /\left|\mathbf{c}_{i}\right|, \quad i=1,2,3, \\
\mathbf{c}_{1} & =\left(\alpha_{2},-\alpha_{1}, 0\right), \\
c_{i} & =\left(\alpha_{1}, \alpha_{2}, \alpha_{3} \frac{\left(v_{p}^{2}-v_{s}^{2}\right)\left(1-\alpha_{3}^{2}\right)}{\left(v_{p}^{2}-v_{3}^{2}\right) \alpha_{3}^{2}+v_{s}^{2}-\lambda_{i}^{2}}\right), \quad i=2,3 .
\end{aligned}
$$

Proof. The proof is based on the laborious task of finding the eigenvalues and the corresponding eigenvectors of the dyadic

$$
\mathbb{M}=\left[\begin{array}{ccc}
\left(v_{p}^{2}-v_{s}^{2}+v_{a}^{2}\right) \alpha_{1}^{2}+v_{s}^{2}+v_{a}^{2} a_{3}^{2} & \left(v_{p}^{2}-v_{s}^{2}+v_{a}^{2}\right) \alpha_{1} \alpha_{2} & \left(v_{p}^{2}-v_{s}^{2}\right) \alpha_{1} \alpha_{3} \\
\left(v_{p}^{2}-v_{s}^{2}+v_{a}^{2}\right) \alpha_{1} \alpha_{2} & \left(v_{p}^{2}-v_{s}^{2}+v_{a}^{2}\right) \alpha_{2}^{2}+v_{s}^{2}+v_{a}^{2} \alpha_{3}^{2} & \left(v_{p}^{2}-v_{s}^{2}\right) \alpha_{2} \alpha_{3} \\
\left(v_{p}^{2}-v_{s}^{2}\right) \alpha_{1} \alpha_{3} & \left(v_{p}^{2}-v_{s}^{2}\right) \alpha_{2} \alpha_{3} & \left(v_{p}^{2}-v_{s}^{2}\right) \alpha_{3}^{2}+v_{s}^{2}
\end{array}\right]
$$

The eigenvalues of $\mathbb{M}$ are the $\lambda_{i}^{2}, i=1,2,3$ and their square roots give the characteristic speeds (phase velocities) of the three waves $\mathbf{w}_{i}, i=1,2,3$. The corresponding eigenvectors $\mathbf{e}_{i}, i=1,2,3$, give the polarization vectors of these waves. Note that the symmetry of $M$ implies that the eigenvalues are real and the eigenvectors are mutually orthogonal. Since $\lambda_{i}^{2}>0, i=1,2,3$, we deduce that all the phase velocities are real. When the constant magnetic field is absent, i.e. when $\mathbf{H}=\mathbf{0}$, then $v_{a}=0$ and Theorem 1 takes the following simple form.

THEOREM $1^{\prime}$. The solution of the system (11) where

$$
M=v_{p}^{2} \alpha \alpha+v_{s}^{2}(0-\alpha \alpha)
$$


that satisfies the initial conditions (16), (17) is given by

$$
\begin{aligned}
\hat{\mathbf{u}}(t)= & \alpha \boldsymbol{\alpha} \cdot\left\lfloor\hat{\mathbf{u}}_{0} \cos \left(\lambda_{1}^{0} r t\right)+\hat{\mathbf{u}}_{1} \frac{\sin \left(\lambda_{1}^{0} r t\right)}{\lambda_{1}^{0} r}\right\rfloor \\
& +(\mathbb{Q}-\boldsymbol{\alpha \alpha}) \cdot\left\lfloor\hat{\mathbf{u}}_{0} \cos \left(\lambda_{2}^{0} r t\right)+\hat{\mathbf{u}}_{1} \frac{\sin \left(\lambda_{2}^{0} r t\right)}{\lambda_{2}^{0} r}\right\rfloor
\end{aligned}
$$

where

$$
\lambda_{1}^{0}=v_{p}, \quad \lambda_{2}^{0}=v_{s} .
$$

Theorem $1^{\prime}$ states that there is a longitudinal wave moving with phase velocity $v_{p}$ and a doubly degenerate transverse wave moving with phase velocity $v_{s}$, i.e. the well-known elastic waves.

The wave $\mathbf{w}_{1}$ is polarized along the $\mathbf{e}_{1}$ direction and since $\mathbf{e}_{1} \cdot \alpha=0$ we conclude that $\mathbf{w}_{1}$ is a transverse wave. The other two waves $\mathbf{w}_{i}, i=2,3$, are polarized along $\mathbf{e}_{i}, i=2,3$ and they are neither transverse nor longitudinal. Therefore, there are three mutually orthogonal polarized plane waves propagating along every direction, each one with different phase velocity, one of them being transverse.

In particular, if the direction of propagation $\alpha$ is orthogonal to the applied constant magnetic field $\mathbf{H}$, i.e. if $\alpha_{3}=0$, then (19)-(21) give

$$
\lambda_{1}^{2}=\lambda_{3}^{2}=v_{s}^{2}, \quad \lambda_{2}^{2}=v_{p}^{2}+v_{a}^{2}
$$

and from (22)-(24) we obtain that

$$
\mathbf{e}_{2}=\alpha
$$

while the $\mathbf{e}_{1}, \mathbf{e}_{3}$ vectors form a plane perpendicular to the direction of propagation. Therefore, for propagation perpendicular to the magnetic field, the $\mathbf{w}_{1}$ and $\mathbf{w}_{3}$ waves are transverse waves, the $\mathbf{w}_{2}$ is a longitudinal wave, and the magnetic field increases only the phase velocity of the longitudinal wave.

For propagation along the direction of the magnetic field, i.e. for $\alpha_{3}=1\left(\alpha_{1}=\alpha_{2}=0\right)$ we obtain

$$
\lambda_{1}^{2}=\lambda_{3}^{2}=v_{s}^{2}+v_{a}^{2}, \quad \lambda_{2}^{2}=v_{p}^{2}
$$

so the polarization vector $\mathbf{e}_{2}$ becomes parallel to the propagation vector $\alpha$, and the other two directions of polarization $\mathbf{e}_{1}, \mathbf{e}_{3}$ form a plane perpendicular to the propagation vector $\alpha$.

Therefore, for propagation along the direction of the magnetic field the $\mathbf{w}_{1}$ and $\mathbf{w}_{3}$ waves are again transverse waves and the $\mathbf{w}_{2}$ wave is longitudinal. The magnetic field, in this case, increases only the phase velocity of the transverse waves.

Summarizing, when the propagation vector is either perpendicular or parallel to the direction of the magnetic field, there are always two transverse and one longitudinal wave, just as in the case where no magnetic field exists. The magnetic field increases the phase velocities of the perpendicularly polarized waves, always by the same amount, proportional to the intensity of the magnetic field.

The above conclusions hold for plane magnetoelastic waves propagating in the particular direction $\alpha$. For the general case we define the $n$-wave by

$$
\mathbf{u}^{n}(\mathbf{x}, t)=\left[\mathbf{w}_{n}(\xi, t)\right]^{\vee}(\mathbf{x}, t), i=1,2,3
$$


where $\mathbf{w}_{n}$ is given by (18) and [ $]^{\vee}$ indicates the inverse Fourier transform operator.

4. Energy theorems. We first prove conservation of energy for the magnetoelastic equation (1)-(2).

THEOREM 2. Let $\mathbf{u}(\mathbf{x}, t)$ be a solution of Eq. (2). If the Cauchy data $\mathbf{u}_{0}, \mathbf{u}_{1}$ correspond to finite energy, i.e. if $\mathbf{u}_{0} \in\left[H^{1}\left(\mathbb{R}^{3}\right)\right]^{3}$ and $\mathbf{u}_{1} \in\left[L^{2}\left(\mathbb{R}^{3}\right)\right]^{3}$, then for each $t>0$ we have

$$
E(t)=\left.\int_{\mathbb{R}^{3}}\left|\frac{\rho}{2}\right| \mathbf{u}_{t}\right|^{2}+\frac{\mu}{2}(\nabla \mathbf{u}: \nabla \mathbf{u})+\frac{\lambda+\mu}{2}|\nabla \cdot \mathbf{u}|^{2}+\frac{\mu_{0}}{8 \pi}|\mathbf{h}|^{2} \mid d^{3} x=E(0),
$$

where

$$
\nabla \mathbf{u}: \nabla \mathbf{u}=\sum_{i, j=L}^{3}\left(\frac{\partial u_{i}}{\partial x_{j}}\right)^{2}
$$

Hence the total energy is conserved.

Remark. The first three terms in the integrand correspond to the sum of the kinetic and the strain energy of elastic waves. The last term is due to the presence of the magnetic field and may be interpreted as an "interaction energy" between elastic and electromagnetic waves.

Proof. Following the standard technique multiplying Eq. (2) by $\mathbf{u}_{t}$ we obtain the well-known identities

$$
\begin{gathered}
\rho \mathbf{u}_{t} \cdot \mathbf{u}_{t t}=\frac{\partial}{\partial t}\left(\frac{\rho}{2}\left|\mathbf{u}_{t}\right|^{2}\right), \\
-\mu \mathbf{u}_{t} \cdot \nabla^{2} \mathbf{u}=-\nabla \cdot\left[\mu(\nabla \mathbf{u}) \cdot \mathbf{u}_{t}\right]+\frac{\partial}{\partial t}\left(\frac{\mu}{2} \nabla \mathbf{u}: \nabla \mathbf{u}\right), \\
-(\lambda+\mu) \mathbf{u}_{t} \cdot \nabla(\nabla \cdot \mathbf{u})=-\nabla \cdot\left[(\lambda+\mu)(\nabla \cdot \mathbf{u}) \mathbf{u}_{t}\right]+\frac{\partial}{\partial t}\left(\frac{\lambda+\mu}{2}|\nabla \cdot \mathbf{u}|^{2}\right) .
\end{gathered}
$$

We also have that

$$
\begin{aligned}
\nabla \cdot\left[\mathbf{h} \times\left(\mathbf{H} \times \mathbf{u}_{t}\right)\right]=\left(\mathbf{H} \times \mathbf{u}_{t}\right) \cdot(\nabla \times \mathbf{h})-\mathbf{h} \cdot \nabla \times\left(\mathbf{H} \times \mathbf{u}_{t}\right) \\
=(\nabla \times \mathbf{h}) \cdot\left(\mathbf{H} \times \mathbf{u}_{t}\right)+\mathbf{h} \cdot \nabla \times\left(\mathbf{u}_{t} \times \mathbf{H}\right) \\
=[(\nabla \times \mathbf{h}) \times \mathbf{H}] \cdot \mathbf{u}_{t}+\mathbf{h} \cdot \frac{\partial}{\partial t}[\nabla \times(\mathbf{u} \times \mathbf{H})]
\end{aligned}
$$

which in view of (1) gives the identity

$$
\begin{aligned}
& -\frac{\mu_{0}}{8 \pi} \mathbf{u}_{t} \cdot[(\nabla \times \mathbf{h}) \times \mathbf{H}] \\
= & -\frac{\mu_{0}}{4 \pi} \nabla \cdot\left[\mathbf{h} \times\left(\mathbf{H} \times \mathbf{u}_{t}\right)\right]+\frac{\partial}{\partial t}\left(\frac{\mu_{0}}{8 \pi}|\mathbf{h}|^{2}\right) .
\end{aligned}
$$

Adding up Eqs. (34), (35), (36) and (38) and using (2), we obtain

$$
\begin{aligned}
\left.\frac{\partial}{\partial t}\left|\frac{\rho}{2}\right| \mathbf{u}_{t}\right|^{2}+\frac{\mu}{2}(\nabla \mathbf{u}: \nabla \mathbf{u})+ & \frac{\lambda+\mu}{2}|\nabla \cdot \mathbf{u}|^{2}+\frac{\mu_{0}}{8 \pi}|\mathbf{h}|^{2} \mid=\frac{\partial}{\partial t} e(t) \\
& =\nabla \cdot\left|\mu(\nabla \mathbf{u}) \cdot \mathbf{u}_{t}+(\lambda+\mu)(\nabla \cdot \mathbf{u}) \mathbf{u}_{t}+\frac{\mu_{0}}{4 \pi} \mathbf{h} \times\left(\mathbf{H} \times \mathbf{u}_{t}\right)\right|
\end{aligned}
$$


where $e(t)$ is the energy density.

Integrating (39) over a sphere of radius $R$, using the Gauss theorem and the fact that for each $t>0, \mathbf{u}(t) \in\left[H^{2}\right]^{3}$ and $\mathbf{u}_{t}(t) \in\left[L^{2}\right]^{3}$, we see that the surface integral vanishes as $R \rightarrow \infty$ and we obtain

$$
\frac{\partial}{\partial t} \int_{\mathbb{R}^{3}} e(t) d^{3} \times=\frac{d}{d t} E(t)=0
$$

or

$$
E(t)=\text { constant }=E(0), \quad t \geq 0
$$

where $E(0)$ is the initial total energy. This shows conservation of energy and completes the proof.

By Parseval's theorem one can evaluate the energy integrals using the Fourier transform of the solution. Eq. (33) takes the form

$$
E(t)=K(t)+S(t)+I(t)=K(0)+S(0)+I(0)=E(0)
$$

where the kinetic energy $K(t)$, the strain energy $S(t)$, and the interaction energy $I(t)$ are given by

$$
\begin{aligned}
K(t) & =\int_{\mathbb{R}^{3}} \frac{\rho}{2}\left|\mathbf{u}_{t}\right|^{2} d^{3} x=\int_{\mathbb{R}^{3}} \frac{\rho}{2}\left|\hat{\mathbf{u}}_{t}\right|^{2} d^{3} \xi, \\
S(t) & \left.=\left.\int_{\mathbb{R}^{3}}\left|\frac{\mu}{2}(\nabla \mathbf{u}: \nabla \mathbf{u})+\frac{\lambda+\mu}{2}\right| \nabla \cdot \mathbf{u}\right|^{2}\right] d^{3} x \\
& =\int_{\mathbb{R}^{3}} \frac{\rho r^{2}}{2}\left[v_{s}^{2}|\hat{\mathbf{u}}|^{2}+\left(v_{p}^{2}-v_{s}^{2}\right)|\alpha \cdot \hat{\mathbf{u}}|^{2}\right] d^{3} \xi, \\
I(t) & =\int_{\mathbb{R}^{3}} \frac{\mu_{0}}{8 \pi}|\mathbf{h}|^{2} d^{3} x \\
& =\int_{\mathbb{R}^{3}} \frac{\rho r^{2} v_{a}^{2}}{2}\left[\alpha_{3}^{2}|\hat{\mathbf{u}}|^{2}+|\alpha \cdot \hat{\mathbf{u}}|^{2}-\left.2 \alpha_{3}^{2} \int \mathbf{x}_{3} \cdot \hat{\mathbf{u}}\right|^{2}\right] d^{3} \xi,
\end{aligned}
$$

respectively. Similarly at $t=0$ we have

$$
\begin{aligned}
& K(0)=\int_{\mathbb{R}^{3}} \frac{\rho}{2}\left|\mathbf{u}_{t}\right|^{2} d^{3} x=\int_{\mathbb{R}^{3}} \frac{\rho}{2}\left|\hat{\mathbf{u}}_{1}\right|^{2} d^{3} \xi \\
& S(0)\left.=\left.\int_{\mathbb{R}^{3}}\left|\frac{\mu}{2}\left(\nabla \mathbf{u}_{0}: \nabla \mathbf{u}_{0}\right)+\frac{\lambda+\mu}{2}\right| \nabla \cdot \mathbf{u}_{0}\right|^{2}\right] d^{3} x \\
&=\int_{\mathbb{R}^{3}} \frac{\rho r^{2}}{2}\left[v_{s}^{2}\left|\hat{\mathbf{u}}_{0}\right|^{2}+\left(v_{p}^{2}-v_{s}^{2}\right)\left|\alpha \cdot \hat{\mathbf{u}}_{0}\right|^{2}\right] d^{3} \xi \\
& I(0)=\int_{\mathbb{R}^{3}} \frac{\mu_{0}}{8 \pi}|\mathbf{h}(\mathbf{x}, 0)|^{2} d^{3} x=\int_{\mathbb{R}^{3}} \frac{\rho r^{2} v_{a}^{2}}{2}\left[\alpha_{3}^{2}\left|\hat{\mathbf{u}}_{0}\right|^{2}+\left|\alpha \cdot \hat{\mathbf{u}}_{0}\right|^{2}-2 \alpha_{3}^{2}\left|\mathbf{x}_{3} \cdot \hat{\mathbf{u}}_{0}\right|^{2}\right] d^{3} \xi .
\end{aligned}
$$

Note that the interaction energy vanishes when $v_{a}=0$ (i.e. $\mathbf{H}=\mathbf{0}$ ). We also define the 
n-kinetic energy by

$$
K_{n}(t)=\int_{\mathbb{R}^{3}} \frac{\rho}{2}\left|\mathbf{u}_{t}^{n}\right|^{2} d^{3} x
$$

the $n$-strain energy by

$$
S_{n}(t)=\int_{\mathbb{R}^{3}}\left[\frac{\mu}{2}\left(\nabla \mathbf{u}^{n}: \nabla \mathbf{u}^{n}\right)+\frac{\lambda+\mu}{2}\left|\nabla \cdot \mathbf{u}^{n}\right|^{2}\right\rfloor d^{3} x,
$$

and the n-interaction energy by

$$
I_{n}(t)=\int_{\mathbb{R}^{3}} \frac{\mu_{0}}{8 \pi}\left|\mathbf{h}^{n}\right| d^{3} x
$$

where

$$
\mathbf{h}^{n}=\nabla \times\left(\mathbf{u}^{n} \times \mathbf{H}\right)
$$

and $\mathbf{u}^{n}$ is given by (32). The n-energy is defined by

$$
E_{n}(t)=K_{n}(t)+S_{n}(t)+I_{n}(t)
$$

then

$$
E(t)=\sum_{n=1}^{3} E_{n}(t)
$$

The next theorem asserts that the total energy of the transverse wave $\mathbf{u}^{1}$ is conserved.

THEOREM 3. Under the hypotheses of Theorem 2

$$
E_{1}(t)=K_{1}(t)+S_{1}(t)+I_{1}(t)=\text { constant, } t \geq 0,
$$

where the subindex 1 indicates that in the formulae $\hat{\mathbf{u}}$ is replaced by $\mathbf{w}_{1}$ as given by (18).

Proof. By Parseval's theorem we can evaluate the energy $E_{1}$ using the Fourier transform $w_{1}$ of $u^{1}$. The transverse wave $w_{1}$ is given by

$$
\mathbf{w}_{1}=\mathbf{e}_{1} \mathbf{e}_{1} \cdot\left\lfloor\hat{\mathbf{u}}_{0} \cos \left(\lambda_{1} r t\right)+\hat{\mathbf{u}}_{1} \frac{\sin \left(\lambda_{1} r t\right)}{\lambda_{1} r}\right\rfloor
$$

where

$$
\lambda_{1}^{2}=v_{s}^{2}+v_{a}^{2} \alpha_{3}^{2}, \quad \mathbf{e}_{1}=\left(\alpha_{1}^{2}+\alpha_{2}^{2}\right)^{-1 / 2}\left(\alpha_{2},-\alpha_{1}, 0\right) .
$$

Since $\alpha \cdot w_{1}=x_{3} \cdot w_{1}=0$ the total energy of $w_{1}$ is given by

$$
E_{1}(t)=\int_{\mathbb{R}^{3}} \frac{\rho}{2}\left[\left|\mathbf{w}_{1 t}\right|^{2}+v_{s}^{2} r^{2}\left|\mathbf{w}_{1}\right|^{2}+v_{a}^{2} r^{2} \alpha_{3}^{2}\left|\mathbf{w}_{1}\right|^{2}\right] d^{3} \xi .
$$

Substituting $w_{1}$ from $(56)$ into (58), we obtain finally that

$$
E_{1}(t)=\int_{\mathbb{R}^{3}} \frac{\rho}{2}\left[\lambda_{1}^{2} r^{2}\left|\mathbf{e}_{1} \cdot \hat{\mathbf{u}}_{0}\right|^{2}+\left|\mathbf{e}_{1} \cdot \hat{\mathbf{u}}_{1}\right|^{2}\right] d^{3} \xi
$$

for each $t \geq 0$. Hence the total energy of the $w_{1}$ wave is independent of time.

Corollary: Under the hypotheses of Theorem 2, the sum of the total energy of the two oblique waves $\mathbf{w}_{2}$ and $\mathbf{w}_{3}$ is conserved. 
Proof. The proof is a straightforward consequence of Theorems 2 and 3.

The following two lemmas are fundamental for the proof of Theorem 4 below.

LEMMA 1 . Let $f: \mathbb{R}^{3} \rightarrow \mathbb{R}$ be an absolutely integrable function of compact support and assume that $f(\mathbf{x})=0,|\mathbf{x}|>R$. Let $\hat{f}: \mathbb{R}^{3} \rightarrow \mathbb{C}$ be the three-dimensional Fourier transform of $f(\mathbf{x})$. Define the iterated integral

$$
\begin{gathered}
C(t)=\int_{\mathbb{R}^{3}}|\hat{f}(\xi)|^{2} \cos (2 t|\xi|) d^{3} \xi=\int_{0}^{\infty} H_{c}(|\xi|) \cos (2 t|\xi|) d|\xi|, \\
H_{c}(|\xi|)=|\xi|^{2} \int_{0}^{2 \pi} d \varphi \int_{0}^{\pi}|\hat{f}|^{2} \sin \theta d \theta,
\end{gathered}
$$

where $(|\xi|, \theta, \varphi)$ are spherical coordinates in the $\xi$-space. Then $H_{c}$ is an even function of $|\xi|$, and $C(t)=0$ for $t \geq R$.

Lemma 2. Let $f, \hat{f}$ be as in Lemma 1 and let $g, \hat{g}$ be another such pair. Define the iterated integral

$$
\begin{gathered}
S(t)=\int_{\mathbb{R}^{3}} \operatorname{Im}\left(\hat{f}^{*}\right)(\xi) \sin (2 t|\xi|) d^{3} \xi=\int_{0}^{\infty} H_{s}(|\xi|) \sin (2 t|\xi|) d|\xi|, \\
H_{s}(|\xi|)=|\xi|^{2} \int_{0}^{2 n} d \varphi \int_{0}^{\pi} \operatorname{Im}\left(\hat{f}^{*}\right) \sin \theta d \theta,
\end{gathered}
$$

where ${ }^{*}$ denotes complex conjugate. Then $H_{s}$ is an odd function of $|\xi|$, and $S(t)=0$ for $t \geq R$.

The proofs of Lemmas 1 and 2 can be found in the proof of Theorem 1 in [5] and are based on the following corollary to the Paley-Wiener theorem.

Proposition. Let $H(z)$ be an entire function of exponential type $c$ which is absolutely integrable on the real line. Then the support of the Fourier transform of $H$ is contained in $[-c,+c]$.

Proof. See [5].

We state and prove next the finite equipartition of energy theorem.

THEOREM 4. Let $\mathbf{u}(\mathbf{x}, t)$ be a solution of Eq. (2) satisfying the initial conditions (14)-(15). Assume that $\mathbf{u}_{0}, \mathbf{u}_{1}$ are infinitely smooth with compact support and that

$$
\text { supp } \mathbf{u}_{0}(\mathbf{x}) \subset S(0 ; R), \quad \operatorname{supp} \mathbf{u}_{1}(\mathbf{x}) \subset S(0 ; R)
$$

where

$$
S(0 ; R)=\left\{\mathbf{x} \in \mathbb{R}^{3}|| \mathbf{x} \mid \leq R\right\} .
$$

Then

$$
K(t)=S(t)+I(t)=\frac{1}{2} E(0), \quad t \geq R / v_{0}
$$

where $K(t)$ is the kinetic energy, $S(t)$ is the strain energy and $I(t)$ the interaction energy given by (43), (44) and (45), respectively, $E(0)$ is the total energy of the initial data and

$$
v_{0}=\min \left\{\lambda_{1}, \lambda_{2}, \lambda_{3},\left|\lambda_{1}-\lambda_{2}\right|,\left|\lambda_{2}-\lambda_{3}\right|,\left|\lambda_{3}-\lambda_{1}\right|\right\} .
$$


Proof. Using (18), we obtain after some tedious calculations the following expressions which contain either terms independent of $t$ or terms that depend on $t$ through sine and cosine:

$$
\begin{aligned}
& \left|\hat{\mathbf{u}}_{t}(t)\right|^{2}=\frac{1}{2}\left|\hat{\mathbf{u}}_{1}\right|^{2}+\frac{r^{2}}{2} \sum_{i=1}^{3} \lambda_{i}^{2}\left|\mathbf{e}_{i} \cdot \hat{\mathbf{u}}_{0}\right|^{2} \\
& \left.+\left.\sum_{i=1}^{3}\left|\frac{1}{2}\right| \mathbf{e}_{i} \cdot \hat{\mathbf{u}}_{1}\right|^{2}-\frac{\lambda_{i}^{2} r^{2}}{2}\left|\mathbf{e}_{i} \cdot \hat{\mathbf{u}}_{0}\right|^{2}\right] \cos \left(2 \lambda_{i} r t\right) \\
& -\sum_{i=1}^{3} \lambda_{i} r \operatorname{Re}\left[\left(\mathbf{e}_{i} \cdot \hat{\mathbf{u}}_{0}\right)\left(\mathbf{e}_{i} \cdot \hat{\mathbf{u}}_{1}^{*}\right)\right] \sin \left(2 \lambda_{i} r t\right), \\
& |\hat{\mathbf{u}}(t)|^{2}=\frac{1}{2}\left|\hat{\mathbf{u}}_{0}\right|^{2}+\frac{1}{2 r^{2}} \sum_{i=1}^{3} \frac{1}{\lambda_{i}^{2}}\left|\mathbf{e}_{i} \cdot \hat{\mathbf{u}}_{1}\right|^{2} \\
& +\left.\sum_{i=1}^{3}\left|\frac{1}{2}\right| \mathbf{e}_{i} \cdot \hat{\mathbf{u}}_{0}\right|^{2}-\frac{1}{2 \lambda_{i}^{2} r^{2}}\left|\mathbf{e}_{i} \cdot \hat{\mathbf{u}}_{1}\right|^{2} \mid \cos \left(2 \lambda_{i} r t\right) \\
& +\sum_{i=1}^{3} \frac{1}{\lambda_{i} r} \operatorname{Re}\left[\left(\mathbf{e}_{i} \cdot \hat{\mathbf{u}}_{0}\right)\left(\mathbf{e}_{i} \cdot \hat{\mathbf{u}}_{1}^{*}\right)\right] \sin \left(2 \lambda_{i} r t\right), \\
& |\alpha \cdot \hat{\mathbf{u}}(t)|^{2}=\frac{1}{2}\left|\alpha \cdot \hat{\mathbf{u}}_{0}\right|^{2}+\frac{1}{2 r^{2}} \sum_{i=1}^{3} \frac{1}{\lambda_{i}^{2}}\left|\alpha \cdot \mathbf{e}_{i}\right|^{2}\left|\mathbf{e}_{i} \cdot \hat{\mathbf{u}}_{1}\right|^{2} \\
& \left.+\left.\frac{1}{2} \sum_{i=1}^{3}\left|\alpha \cdot \mathbf{e}_{i}\right|^{2}|| \mathbf{e}_{i} \cdot \hat{\mathbf{u}}_{0}\right|^{2}-\frac{1}{\lambda_{i}^{2} r^{2}}\left|\mathbf{e}_{i} \cdot \hat{\mathbf{u}}_{1}\right|^{2}\right] \cos \left(2 \lambda_{i} r t\right) \\
& +\frac{1}{r} \sum_{i=1}^{3} \frac{\left|\alpha \cdot \mathbf{e}_{i}\right|^{2}}{\lambda_{i}} \operatorname{Re}\left[\left(\mathbf{e}_{i} \cdot \hat{\mathbf{u}}_{0}\right)\left(\mathbf{e}_{i} \cdot \hat{\mathbf{u}}_{1}\right)^{*}\right] \sin \left(2 \lambda_{i} r t\right) \\
& +\frac{1}{2} \sum_{\substack{i, j=1 \\
i \neq j}}^{3}\left(\alpha \cdot \mathbf{e}_{i}\right)\left(\alpha \cdot \mathbf{e}_{j}\right) \\
& \cdot\left\{\left(\mathbf{e}_{i} \cdot \hat{\mathbf{u}}_{o}\right)\left(\mathbf{e}_{j} \cdot \hat{\mathbf{u}}_{0}\right) *\left[\cos \left(\left(\lambda_{i}+\lambda_{j}\right) r t\right)+\cos \left(\left(\lambda_{i}-\lambda_{j}\right) r t\right)\right]\right. \\
& +\frac{\left(\mathbf{e}_{i} \cdot \hat{\mathbf{u}}_{1}\right)\left(\mathbf{e}_{j} \cdot \hat{\mathbf{u}}_{1}\right)^{*}}{\lambda_{i} \lambda_{j} r^{2}}\left[\cos \left(\left(\lambda_{i}-\lambda_{j}\right) r t\right)-\cos \left(\left(\lambda_{i}+\lambda_{j}\right) r t\right)\right] \\
& +\frac{2}{\lambda_{i} r} \operatorname{Re}\left(\left(\mathbf{e}_{i} \cdot \hat{\mathbf{u}}_{0}\right)\left(\mathbf{e}_{j} \cdot \hat{\mathbf{u}}_{1}\right)^{*}\right)\left[\sin \left(\left(\lambda_{i}+\lambda_{j}\right) r t\right)+\sin \left(\left(\lambda_{i}-\lambda_{j}\right) r t\right)\right] \text {, } \\
& \left|\mathbf{x}_{3} \cdot \hat{\mathbf{u}}\right|^{2}=\frac{1}{2}\left|\mathbf{x}_{3} \cdot \hat{\mathbf{u}}_{0}\right|^{2}+\frac{1}{2 r^{2}} \sum_{i=2}^{3} \frac{1}{\lambda_{i}^{2}}\left|\mathbf{x}_{3} \cdot \mathbf{e}_{i}\right|^{2}\left|\mathbf{e}_{i} \cdot \hat{\mathbf{u}}_{1}\right|^{2} \\
& \left.+\left.\frac{1}{2} \sum_{i=2}^{3}\left|\mathbf{x}_{3} \cdot \mathbf{e}_{i}\right|^{2}|| \mathbf{e}_{i} \cdot \hat{\mathbf{u}}_{0}\right|^{2}-\frac{1}{\lambda_{i}^{2} r^{2}}\left|\mathbf{e}_{i} \cdot \hat{\mathbf{u}}_{1}\right|^{2}\right] \cos \left(2 \lambda_{i} r t\right) \\
& +\frac{1}{r} \sum_{i=2}^{3} \frac{\left|\mathbf{x}_{3} \cdot \boldsymbol{e}_{i}\right|^{2}}{\lambda_{i}} \operatorname{Re}\left[\left(\mathbf{e}_{i} \cdot \hat{\mathbf{u}}_{0}\right)\left(\mathbf{e}_{i} \cdot \hat{\mathbf{u}}_{1}\right)^{*}\right] \sin \left(2 \lambda_{i} r t\right) \\
& +\frac{1}{2} \sum_{\substack{i, j=2 \\
i \neq j}}^{3}\left(\mathbf{x}_{3} \cdot \mathbf{e}_{i}\right)\left(\mathbf{x}_{3} \cdot \mathbf{e}_{j}\right)
\end{aligned}
$$




$$
\begin{aligned}
& \cdot\left\{\left(\mathbf{e}_{i} \cdot \hat{\mathbf{u}}_{0}\right)\left(\mathbf{e}_{j} \cdot \hat{\mathbf{u}}_{0}\right)^{*}\left[\cos \left(\left(\lambda_{i}+\lambda_{j}\right) r t\right)+\cos \left(\left(\lambda_{i}-\lambda_{j}\right) r t\right)\right]\right. \\
& +\frac{\left(\mathbf{e}_{i} \cdot \hat{\mathbf{u}}_{1}\right)\left(\mathbf{e}_{j} \cdot \hat{\mathbf{u}}_{1}\right)^{*}}{\lambda_{i} \lambda_{j} r^{2}}\left[\cos \left(\left(\lambda_{i}-\lambda_{j}\right) r t\right)-\cos \left(\left(\lambda_{i}+\lambda_{j}\right) r t\right)\right] \\
& \left.\quad+\frac{2}{\lambda_{i} r} \operatorname{Re}\left(\left(\mathbf{e}_{i} \cdot \hat{\mathbf{u}}_{0}\right)\left(\mathbf{e}_{j} \cdot \hat{\mathbf{u}}_{1}\right)^{*}\right)\left[\sin \left(\left(\lambda_{i}+\lambda_{j}\right) r t\right)+\sin \left(\left(\lambda_{i}-\lambda_{j}\right) r t\right)\right]\right\}
\end{aligned}
$$

Substituting (67), (68), (69), (70) in (43), (44), (45) and applying Lemma 1 to terms that are proportional to cosine and Lemma 2 to terms that are proportional to sine, we obtain for each $t \geq R / v_{0}$ the following:

$$
\begin{gathered}
K(t)=\left.\frac{1}{2} \int_{\mathbb{R}^{3}} \frac{\rho}{2}|| \hat{\mathbf{u}}_{1}\right|^{2}+r^{2} \sum_{i=1}^{3} \lambda_{i}^{2}\left|\mathbf{e}_{i} \cdot \hat{\mathbf{u}}_{0}\right|^{2} \mid d^{3} \xi, \\
S(t)=\left.\frac{1}{2} \int_{\mathbb{R}^{3}} \frac{\rho r^{2}}{2}\left|v_{s}^{2}\right| \hat{\mathbf{u}}_{0}\right|^{2}+\frac{v_{s}^{2}}{r^{2}} \sum_{i=1}^{3} \frac{\left|\mathbf{e}_{i} \cdot \hat{\mathbf{u}}_{1}\right|^{2}}{\lambda_{i}^{2}} \\
+\left(v_{p}^{2}-v_{s}^{2}\right)\left|\alpha \cdot \hat{\mathbf{u}}_{0}\right|^{2}+\frac{v_{p}^{2}-v_{s}^{2}}{r^{2}} \sum_{i=1}^{3} \frac{\left|\alpha \cdot \mathbf{e}_{i}\right|^{2}\left|\mathbf{e}_{i} \cdot \hat{\mathbf{u}}_{1}\right|^{2}}{\lambda_{i}^{2}} \mid d^{3} \xi, \\
I(t)=\left.\frac{1}{2} \int_{\mathbb{R}^{3}} \frac{\rho r^{2} v_{a}^{2}}{2}\left|\alpha_{3}^{2}\right| \hat{\mathbf{u}}_{0}\right|^{2}+\frac{\alpha_{3}^{2}}{r^{2}} \sum_{i=1}^{3} \frac{\left|\mathbf{e}_{i} \cdot \hat{\mathbf{u}}_{1}\right|^{2}}{\lambda_{i}^{2}} \\
+\left|\alpha \cdot \hat{\mathbf{u}}_{0}\right|^{2}+\frac{1}{r^{2}} \sum_{i=1}^{3} \frac{\left|\alpha \cdot \mathbf{e}_{i}\right|^{2}\left|\mathbf{e}_{i} \cdot \hat{\mathbf{u}}_{1}\right|^{2}}{\lambda_{i}^{2}} \\
-2 \alpha_{3}^{2}\left|\mathbf{x}_{3} \cdot \hat{\mathbf{u}}_{0}\right|^{2}-\frac{2 \alpha_{3}^{2}}{r^{2}} \sum_{i=2}^{3} \frac{\left|\mathbf{x}_{3} \cdot \mathbf{e}_{i}\right|^{2}\left|\mathbf{e}_{i} \cdot \hat{\mathbf{u}}_{1}\right|^{2}}{\lambda_{i}^{2}} \mid d^{3} \xi .
\end{gathered}
$$

If we set

$$
\begin{gathered}
\left.A_{K}^{0}=\left.\int_{\mathbb{R}^{3}} \frac{\rho}{2} r^{2}\left|\sum_{i=1}^{3} \lambda_{i}^{2}\right| \mathbf{e}_{i} \cdot \hat{\mathbf{u}}_{0}\right|^{2}\right] d^{3} \xi, \\
A_{S}^{1}=\int_{\mathbb{R}^{3}} \frac{\rho}{2}\left[v_{s}^{2} \sum_{i=1}^{3} \frac{\left|\mathbf{e}_{i} \cdot \hat{\mathbf{u}}_{1}\right|^{2}}{\lambda_{i}^{2}}+\left(v_{p}^{2}-v_{s}^{2}\right) \sum_{i=1}^{3} \frac{\left|\alpha \cdot \mathbf{e}_{i}\right|^{2}\left|\mathbf{e}_{i} \cdot \hat{\mathbf{u}}_{1}\right|^{2}}{\lambda_{i}^{2}}\right] d^{3} \xi, \\
A_{I}^{1}=\int_{\mathbb{R}^{3}} \frac{\rho v_{a}^{2}}{2} \mid \alpha_{3}^{2} \sum_{i=1}^{3} \frac{\left|\mathbf{e}_{i} \cdot \hat{\mathbf{u}}_{1}\right|^{2}}{\lambda_{i}^{2}}+\sum_{i=1}^{3} \frac{\left|\alpha \cdot \mathbf{e}_{i}\right|^{2}\left|\mathbf{e}_{i} \cdot \hat{\mathbf{u}}_{1}\right|^{2}}{\lambda_{i}^{2}} \\
-2 \alpha_{3}^{2} \sum_{i=2}^{3} \frac{\left|\mathbf{x}_{3} \cdot \mathbf{e}_{i}\right|^{2}\left|\mathbf{e}_{i} \cdot \hat{\mathbf{u}}_{1}\right|^{2}}{\lambda_{i}^{2}} \mid d^{3} \xi,
\end{gathered}
$$

then

$$
\begin{aligned}
K(t) & =\frac{1}{2} K(0)+\frac{1}{2} A_{K}^{0}, \\
S(t) & =\frac{1}{2} S(0)+\frac{1}{2} A_{S}^{1}, \\
I(t) & =\frac{1}{2} I(0)+\frac{1}{2} A_{I}^{1},
\end{aligned}
$$

where $K(0), S(0)$ and $I(0)$ are given by (46), (47) and (48), respectively. The indices 0,1 in $A_{K}^{0}, A_{S}^{1}, A_{I}^{1}$ indicate that $A_{K}^{0}$ depends on $\hat{\mathbf{u}}_{0}$ and $A_{S}^{1}, A_{I}^{1}$ on $\hat{\mathbf{u}}_{1}$. 
If we add Eqs. (77), (78) and (79) we obtain

$$
K(t)+S(t)+I(t)=\frac{1}{2}(K(0)+S(0)+I(0))+\frac{1}{2}\left(A_{K}^{0}+A_{S}^{1}+A_{I}^{1}\right)
$$

which in view of (42) becomes

$$
E(0)=\frac{1}{2} E(0)+\frac{1}{2}\left(A_{K}^{0}+A_{S}^{1}+A_{l}^{1}\right)
$$

or

$$
A_{K}^{0}+A_{S}^{1}+A_{l}^{1}=E(0)
$$

Therefore we have

$$
K^{1}(0)+S^{0}(0)+I^{0}(0)=A_{K}^{0}+A_{S}^{1}+A_{I}^{1},
$$

where $K^{1}(0), S^{0}(0), I^{0}(0)$ equal $K(0), S(0), I(0)$ respectively, the indices 1,0 indicate the dependence of $K^{1}(0)$ on $\hat{\mathbf{u}}_{1}$ and the dependence of $S^{0}(0), I^{0}(0)$ on $\hat{\mathbf{u}}_{0}$. The relation (83) can be written as

$$
S^{0}(0)+I^{0}(0)-A_{K}^{0}=A_{S}^{1}+A_{I}^{1}-K^{1}(0)
$$

where the left-hand side of $(84)$ depends on $\hat{\mathbf{u}}_{0}$ and the right-hand side depends on $\hat{\mathbf{u}}_{1}$. Hence both sides of (84) equal to the same constant which is independent of $\hat{\mathbf{u}}_{0}$ and $\hat{\mathbf{u}}_{1}$. By choosing $\hat{\mathbf{u}}_{0}=\mathbf{0}$ the above constant is shown to be zero. Therefore,

$$
\begin{gathered}
A_{K}^{0}=S^{0}(0)+I^{0}(0), \\
A_{S}^{1}+A_{I}^{1}=K^{1}(0) .
\end{gathered}
$$

From (77), (78), (79), (82), (85) and (86) we finally obtain for each $t \geq R / v_{0}$

$$
K(t)=\frac{1}{2} K(0)+\frac{1}{2}(S(0)+I(0))=\frac{1}{2} E(0)
$$

and

$$
S(t)+I(t)=\frac{1}{2}(S(0)+I(0))+\frac{1}{2} K(0)=\frac{1}{2} E(0)
$$

which give the relation (65). This completes the proof of the theorem.

The next theorem states the corresponding asymptotic equipartition of energy.

THEOREM 5. Let $\mathbf{u}$ be as in Theorem 4 and assume that $\mathbf{u}_{0} \in\left[H^{1}\left(\mathbb{R}^{3}\right)\right]^{3}$ and $\mathbf{u}_{1} \in\left[L^{2}\left(\mathbb{R}^{3}\right)\right]^{3}$. Then

$$
\lim _{t \rightarrow+\infty} K(t)=\lim _{t \rightarrow+\infty}(S(t)+I(t))=\frac{1}{2} E(0) .
$$

Proof. The proof is the same as the proof of Theorem 4. The time-dependent terms in the expressions for $K(t), S(t)$ and $I(t)$ tend to zero as $t \rightarrow+\infty$ by the Riemann-Lebesgue lemma.

THEOREM 6. Under the hypotheses of Theorem 4 it is true that

$$
K_{1}(t)=S_{1}(t)+I_{1}(t)=\frac{1}{2} E_{1}(0), \quad t \geq R / \lambda_{1}
$$

where $K_{1}, S_{1}, I_{1}$ and $E_{1}$ are given by (49), (50), (51) and (53), respectively.

Proof. Substituting (56) in the expressions (49), (50) and (51) and using Lemmas 1 
and 2 we obtain for $t \geq R / \lambda_{1}$

$$
\begin{aligned}
K_{1}(t) & =\frac{1}{2} \int_{\mathbb{R}^{3}} \frac{\rho}{2}\left[\lambda_{1}^{2} r^{2}\left|\mathbf{e}_{1} \cdot \hat{\mathbf{u}}_{0}\right|^{2}+\left|\mathbf{e}_{1} \cdot \hat{\mathbf{u}}_{1}\right|^{2}\right] d^{3} \xi, \\
S_{1}(t) & =\left.\frac{1}{2} \int_{\mathbb{R}^{3}} \frac{\rho r^{2}}{2}\left|v_{s}^{2}\right| \mathbf{e}_{1} \cdot \hat{\mathbf{u}}_{0}\right|^{2}+\frac{v_{s}^{2}}{\lambda_{1}^{2} r^{2}}\left|\mathbf{e}_{1} \cdot \hat{\mathbf{u}}_{1}\right|^{2} \mid d^{3} \xi, \\
I_{1}(t) & =\left.\frac{1}{2} \int_{\mathbb{R}^{3}} \frac{\rho r^{2} v_{a}^{2}}{2}\left|\alpha_{3}^{2}\right| \mathbf{e}_{1} \cdot \hat{\mathbf{u}}_{0}\right|^{2}+\frac{\alpha_{3}^{2}}{\lambda_{1}^{2} r^{2}}\left|\mathbf{e}_{1} \cdot \hat{\mathbf{u}}_{1}\right|^{2} \mid d^{3} \xi ;
\end{aligned}
$$

or, in view of (19) and (59), we obtain (90).

Theorem 6 asserts that the transverse wave $\mathbf{u}^{1}$, whose total energy is conserved (Theorem 3), attains equipartion between its kinetic and strain plus interaction energy for $t \geq R / \lambda_{1}$.

Theorem 7 below is the asymptotic analogue of Theorem 5 , restricted to the transverse wave $\mathbf{u}^{1}$.

THEOREM 7. Under the hypotheses of Theorem 5 it is true that

$$
\lim _{t \rightarrow+\infty} K_{1}(t)=\lim _{t \rightarrow+\infty}\left(S_{1}(t)+I_{1}(t)\right)=\frac{1}{2} E_{1}(0)
$$

Proof. Use, in place of Lemmas 1 and 2, the Riemann-Lebesgue lemma.

\section{REFERENCES}

[1] D. G. Costa, On partition of energy for uniformly propagative systems, J.M.A.A. 58, 56-62 (1977)

[2] G. Dassios, Equipartition of energy for Maxwells equations, Quart. Appl. Math. (to appear)

[3] G. Dassios, Equipartition of energy in elastic wave propagation, Mechanics Research Communications 6, 45-50 (1979)

[4] G. Dassios and E. Galanis, Asymptotic equipartition of kinetic and strain energy for elastic waves in anisotropic media, Quart. Appl. Math. 38, 121-128 (1980)

[5] R. S. Duffin, Equipartition of energy in wave motion, J.M.A.A. 32, 386-391 (1970)

[6] J. A. Goldstein, An asymptotic property of solutions of wave equations, Proc. A.M.S. 23, 359-363 (1969)

[7] J. A. Goldstein, An asymptotic property of solutions of wave equations II, J.M.A.A. 32, 392-399 (1970)

[8] P. D. Lax and R. S. Phillips, Scattering theory, Academic Press, New York (1967)

[9] H. A. Levine, An equipartition of energy theorem for weak solutions of evolutionary equations in Hilbert space: the Lagrange identity method, J. Diff. Eqs. 24, 197-210 (1977)

[10] W. Nowacki, Dynamic problems of thermoelasticity, Noordhoff, Netherlands (1975)

[11] W. A. Strauss, Some energy components tend to zero globally, Notices A.M.S. 26, *79T-B118, p. A-431 (1979) 